\title{
ANÁLISIS ESPACIAL DE LA VULNERABILIDAD A LA CONTAMINACIÓN DEL AGUA SUBTERRÁNEA DEL ACUÍFERO ASIA-OMAS
}

\author{
SPATIAL ANALYSIS OF THE VULNERABILITY OF POLLUTION GROUNDWATER \\ AQUIFER ASIA-OMAS
}

${ }^{1}$ Jesús Pulcha H. y ${ }^{2}$ Wilfredo Baldeón Q.

\begin{abstract}
Resumen
En el presente trabajo se determinaron la Vulnerabilidad Intrínseca, la Vulnerabilidad a los pesticidas y la vulnerabilidad por intrusión salina en el área de estudio del acuífero Asia-Omas, empleándose las metodologías del Índice DRASTIC, y sus variantes Drastic Pesticida y Drastic Sea-DIndex respectivamente. En la determinación de la Vulnerabilidad intrínseca se identificaron zonas con moderada y alta vulnerabilidad, que representan el 34.39\% del área de estudio, que se ubican en la parte media y baja del acuífero. Los parámetros que tuvieron mayor influencia fueron las variables de profundidad del agua subterránea, recarga y zona vadosa. En cuanto a la vulnerabilidad a la contaminación por Pesticidas se identificaron zonas con moderada vulnerabilidad como las de mayor riesgo, que representan un $21.29 \%$ del área de estudio, asimismo se clasificó en el rango de vulnerabilidad muy baja al 41.24\% del área del acuífero. Respecto a la determinación de la Vulnerabilidad a la contaminación por agua de mar por el proceso de intrusión salina, las categorías con mayor área fueron las de vulnerabilidades baja a despreciable, representando un 79.05\% del área de estudio, mientras que las vulnerabilidades moderada y alta representan el $20.95 \%$.Las variables que tuvieron mayor influencia fueron distancia a línea de costa y conductividad hidráulica.
\end{abstract}

Palabras clave: Vulnerabilidad intrínseca, Índice DRASTIC, contaminación de acuíferos.

\begin{abstract}
In this paper the intrinsic vulnerability, the vulnerability to pesticides and vulnerability by saline intrusion in the study area aquifer Asia-Omas were determined, using the DRASTIC index methodologies and their variants and Pesticide Drastic Drastic Sea-dindex respectively. In determining the intrinsic vulnerability areas with moderate and high vulnerability, representing $34.39 \%$ of the study area, which are located in the middle and lower aquifer were identified. The parameters that were most influential variables were the depth of groundwater recharge and vadosezone. In terms of vulnerability to contamination by pesticides regions with moderate vulnerability as higher risk, representing $21.29 \%$ of the study area also were classified in the range of vulnerability very low at $41.24 \%$ of the aquifer area were identified. Regarding the determination of vulnerability to contamination by sea water by the process of saline intrusion, the categories with the largest area were low to negligible vulnerabilities, representing $79.05 \%$ of the study area, while moderate and high vulnerability representing $20.95 \%$. The variables that had the greatest influence were distance to waterfront and hydraulic conductivity.
\end{abstract}

Key words: intrinsic vulnerability, DRASTIC index, contamination of aquifers.

\section{Introducción}

Actualmente en el País, los acuíferos costeros son altamente sensibles a la sobreexplotación, debido al crecimiento de la demanda poblacional y del sector agrícola. Este es el caso del acuífero Asia-Omas, que en los últimos años ha experimentado un aumento en la demanda hídrica, generando la aparición de procesos de salinización en diferentes sectores del mismo, y de contaminación de sus aguas por las distintas actividades antropogénicas. Una vez contaminado el acuífero, su recuperación es difícil y en muchos casos prácticamente imposible por el costo y tiempo que toma su limpieza, lo cual podría alcanzar décadas o siglos (Losilla et al. 2001).

El objetivo del presente trabajo fue determinar la Vulnerabilidad Intrínseca a la Contaminación por Agua de Mar debido al Proceso de Intrusión Salina, y la Vulnerabilidad a la Contaminación por Pesticidas en el área de estudio. Para ello se empleó la metodología del Índice DRASTIC (Aller et al, 1987), y sus variantes Drastic Sea-DIndex (Bocanegra, 2004) y Drastic Pesticida respectivamente. Este índice es patrocinada por la Agencia de Protección Ambiental de los Estados Unidos (EPA, 1991). La valoración de los parámetros permite acotar los intervalos de vulnerabilidad a la contaminación y delimitar áreas de mayor riesgo frente a un contaminante potencial (Bereciartua, 2003), siendolos resultados un instrumento para la gestión Integral de Recursos Hídricos y Ordenamiento territorial en la zona. Para el desarrollo de esta metodología se hizo uso de la herramienta tecnológica de los Sistemas de Información Geográfica aplicándose la superposición cartográfica mediante el álgebra de mapas. 


\section{Materiales y métodos}

\subsection{Descripción del área de estudio.}

El área de estudio comprende la zona costanera en el límite con el litoral y el sector Esquina de Asia, aproximadamente una distancia de $6 \mathrm{~km}$, en un área aproximada de $46.96 \mathrm{~km} 2$, perteneciente al distrito de Asia, provincia y departamento de Lima, que representa el $65.26 \%$ del área total del acuífero Asia-Omas. Siguiendo la carretera Panamericana Sur se localiza aproximadamente a 102 kilómetros al Sur Este de la ciudad de Lima. Cabe indicar que la zona de trabajo, corresponde al área del estudio "Evaluación de las aguas subterráneas del acuífero Asia-Omas". Este estudio fue realizado por la Autoridad Nacional de Aguaen 2011 y se basa en la caracterización hidrogeoquímica del acuífero (ANA, 2011). Esta zona ha sido seleccionada debido a que existe una demanda creciente de agua y la recarga de la zona es baja por lo que estos estos dos factores hacen que esta parte del acuífero sea altamente Vulnerable a la contaminación y a la intrusión salina.

\subsection{Determinación de los parámetros del Índice DRASTIC}

Para determinar la vulnerabilidad intrínseca del acuífero Asia-Omas, se utilizó el índice DRASTIC. Los 7 parámetros hidrogeológicos que permiten obtener el índice DRASTIC son los siguientes:

1) $\mathrm{D}=$ Depthtowater(profundidad hasta el nivel freático)

2) $\mathrm{R}=$ Recharge(recarga neta)
3) $\mathrm{A}=$ Aquifer media (Medio del acuífero)

4) $\mathrm{S}=$ Soil media (tipo de suelo)

5) $\mathrm{T}=$ Topography(topografía, pendiente)

6) I = Impact of thevadosezone media (impacto del tipo de zona vadosa)

7) $\mathrm{C}=$ Conductivityoftheaquifer(conductividadhidráulica) La profundidad del nivel aguasubterráneo se obtuvo mediante la georeferenciación y digitalización Cartográfica del Mapa de Isoprofundidad elaborado por la ANA para los meses de Mayo a Junio del 2010; posteriormente se creó una red irregular de triangulación con el sistema de información geográfica para posteriormente generar una superficie continua (raster), la cual nos permitió reclasificar los valores de profundidad según los puntajes del índice Drastic.

En la tabla 1 y Fig. 1, se muestran el puntaje asignado y la variación espacial de isoprofundidad del nivel freático del acuífero.

Tabla 1. Reclasificación de Valores de Isoprofundidad.

\begin{tabular}{cc}
\hline Nivel de Profundidad & Puntaje \\
\hline $0-1.5$ & 10 \\
$1.5-9$ & 9 \\
$9-15$ & 7 \\
$15-23$ & 5 \\
$23-30$ & 3 \\
\hline $30-35$ & 1 \\
\hline
\end{tabular}

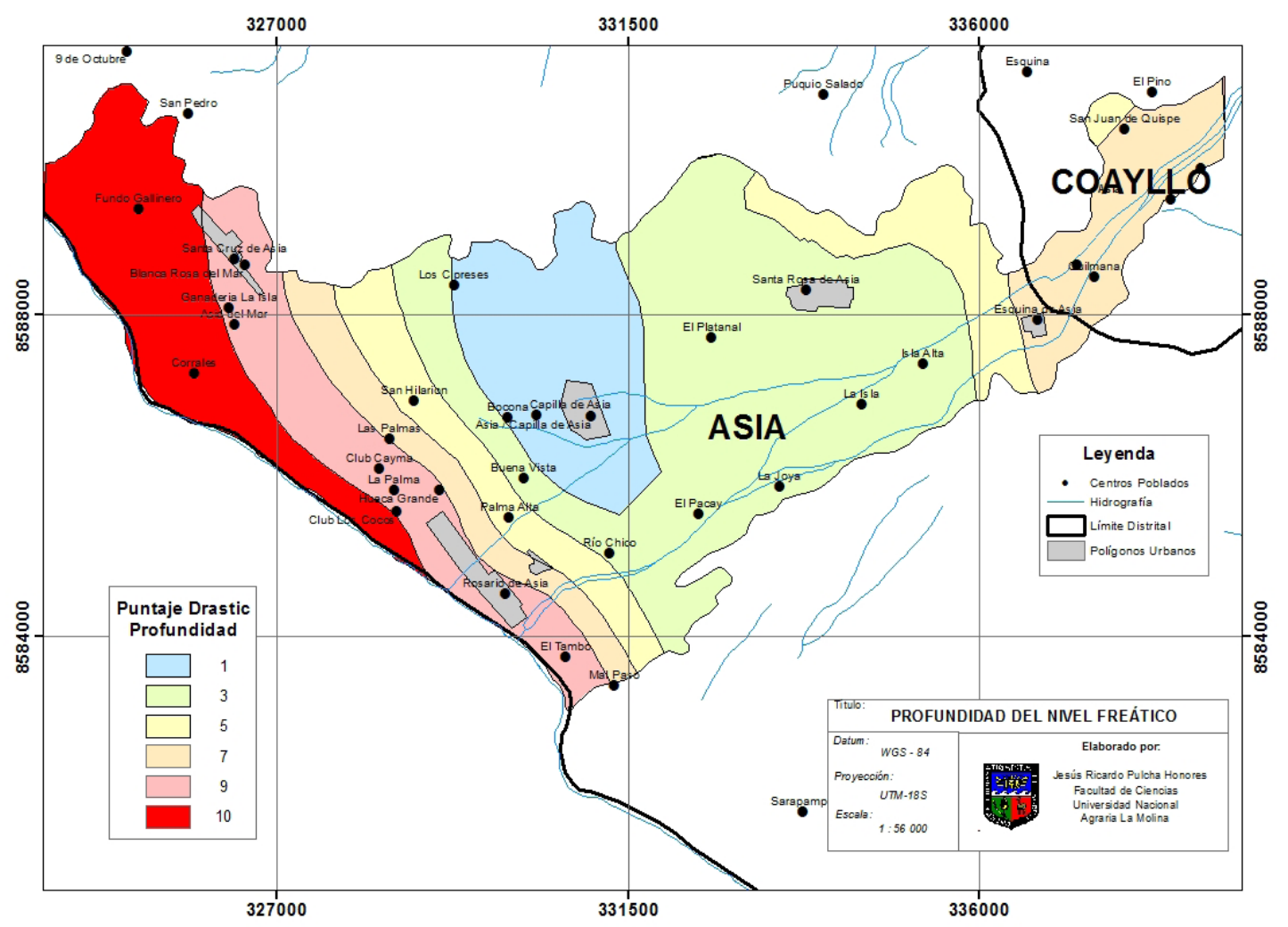

Figura 1. Reclasificación Espacial de Valores de Profundidad del Nivel Freático. 
ANA (2011) determina que en el área de estudio hay escazas precipitaciones, por lo que podemos inferir que la recarga en el área de estudio es muy baja, por lo que se considerócolocar elpuntajede 1 para la recarga (mínima vulnerabilidad o riesgo). Cabe indicar que no se considera las recargas por riego $u$ otra fuente artificial ya que el Índice Drastic evalúa la vulnerabilidad intrínseca o natural del área de estudio.

Para la caracterización de la litología del acuífero se realizó la georeferenciación y digitalización de la Carta Geológica Nacional del Instituto Geológico Minero Metalúrgico INGEMMET hoja 26-n correspondiente a la zona estudio del acuífero (INGEMMET, 1993). En la tabla 2 y Fig. 2 se muestran las regiones geológicas y su respectiva puntuación y variación espacial de dichas regiones ecológicas.

Tabla 2. Reclasificación de Valores de Geología del Acuífero.

\begin{tabular}{cc}
\hline Geología & Puntaje \\
\hline Depósitos Marinos (Qr-m) & 8 \\
Depósitos Aluviales (Qr-al ) & 6 \\
Grupo del Morro del Solar (Ki -ms). & 4 \\
Depósitos Eólicos (Qr-e) & 3 \\
\hline
\end{tabular}

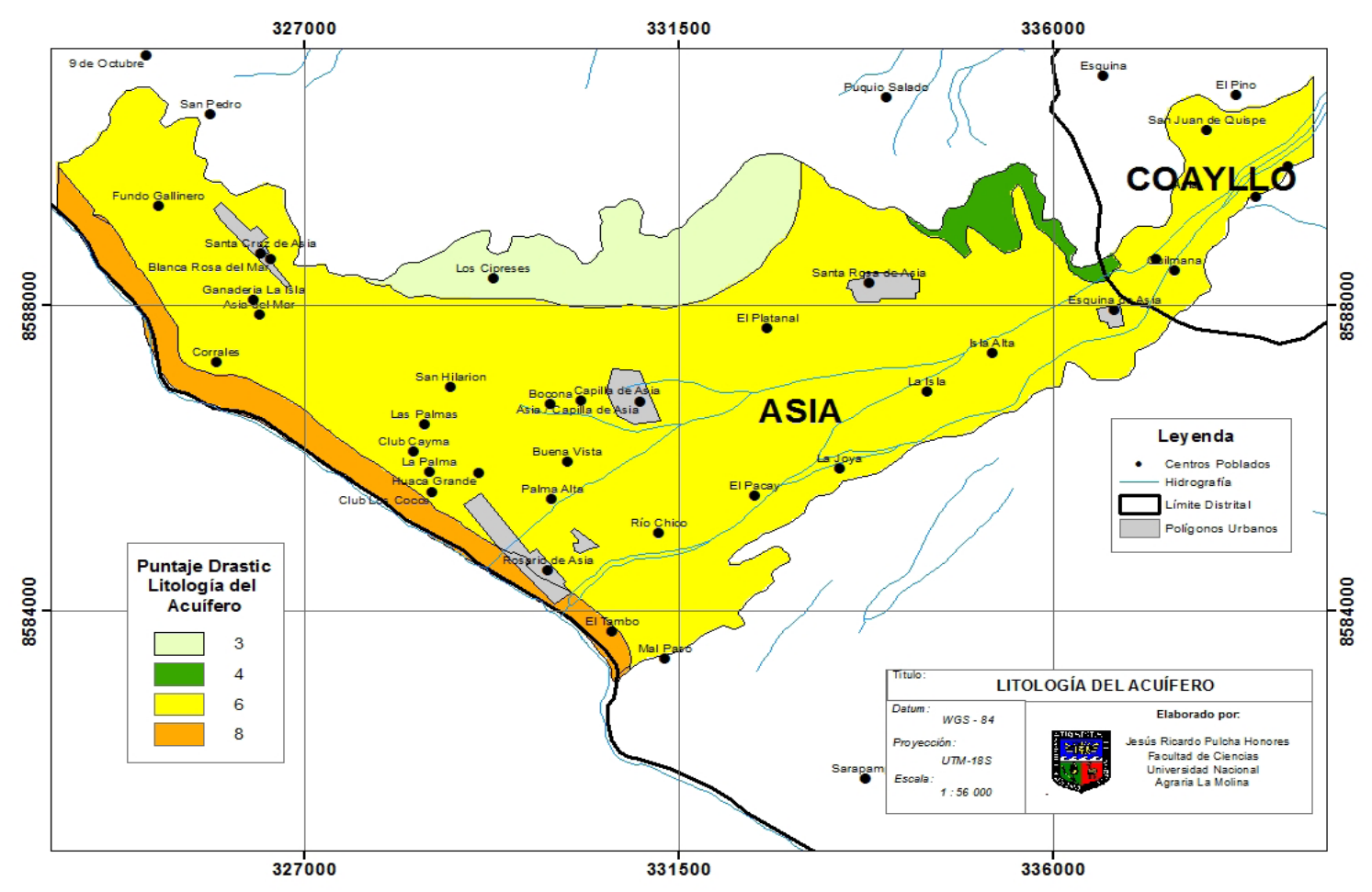

Figura 2. Reclasificación Espacial de Valores de Litología del Acuífero.

Ante la ausencia de cartografía edáfica en el área de estudio, se utilizó los resultados de los perfiles litológicos IRHS $\mathrm{N}^{\circ} 15 / 05 / 02$ - 50 y IRHS $\mathrm{N}^{\circ} 15 / 05 / 02$ - 66 realizados por la ANA en el año 2002 (ANA, 2002), cuya evaluación determina un tipo de suelo arcillolimoso. Por lo que se consideró este resultado y se generalizó para toda el área de estudio del acuífero, fijándose una vulnerabilidad intermedia o riesgo igual a 4 .

Para la elaboración del mapa de pendientes, se utilizó la versión digital de las curvas de nivel de las Cartas Nacionales 26j y 26k del Instituto Geográfico Nacional (IGN). Se generó la Red Irregular de Triangulación (TIN), el Modelo Digital de Elevación (MDE), para finalmente generar el mapa de pendientes, expresado en porcentajes. En la tabla 3 y Fig. 3 se muestra los rangos de pendiente con su puntuación y la variación espacial de la pendiente en el acuífero respectivamente.
Tabla 3. Reclasificación y puntuación de la pendiente del acuífero.

\begin{tabular}{cc}
\hline Rango & Puntaje \\
\hline $0-2$ & 10 \\
$2-6$ & 9 \\
$6-12$ & 5 \\
$12-18$ & 3 \\
$>18$ & 1 \\
\hline
\end{tabular}

De acuerdo a ANA (2002) los resultados de los perfiles litológicosIRHS $\mathrm{N}^{\circ}$ 15/05/02 - 50 y IRHS $\mathrm{N}^{\circ} 15 / 05 / 02$ 66 identifican que la zona vadosa del primer perfil es del tipo Arcilla Limo + Arena Gruesa y la del segundo perfil es del tipo Grava + Arena Arcilla. Por tanto se consideró este resultado y se generalizó para toda el área de estudio del acuífero, fijándose un puntaje Drastic de intermedia vulnerabilidad intrínseca igual a 6 . 


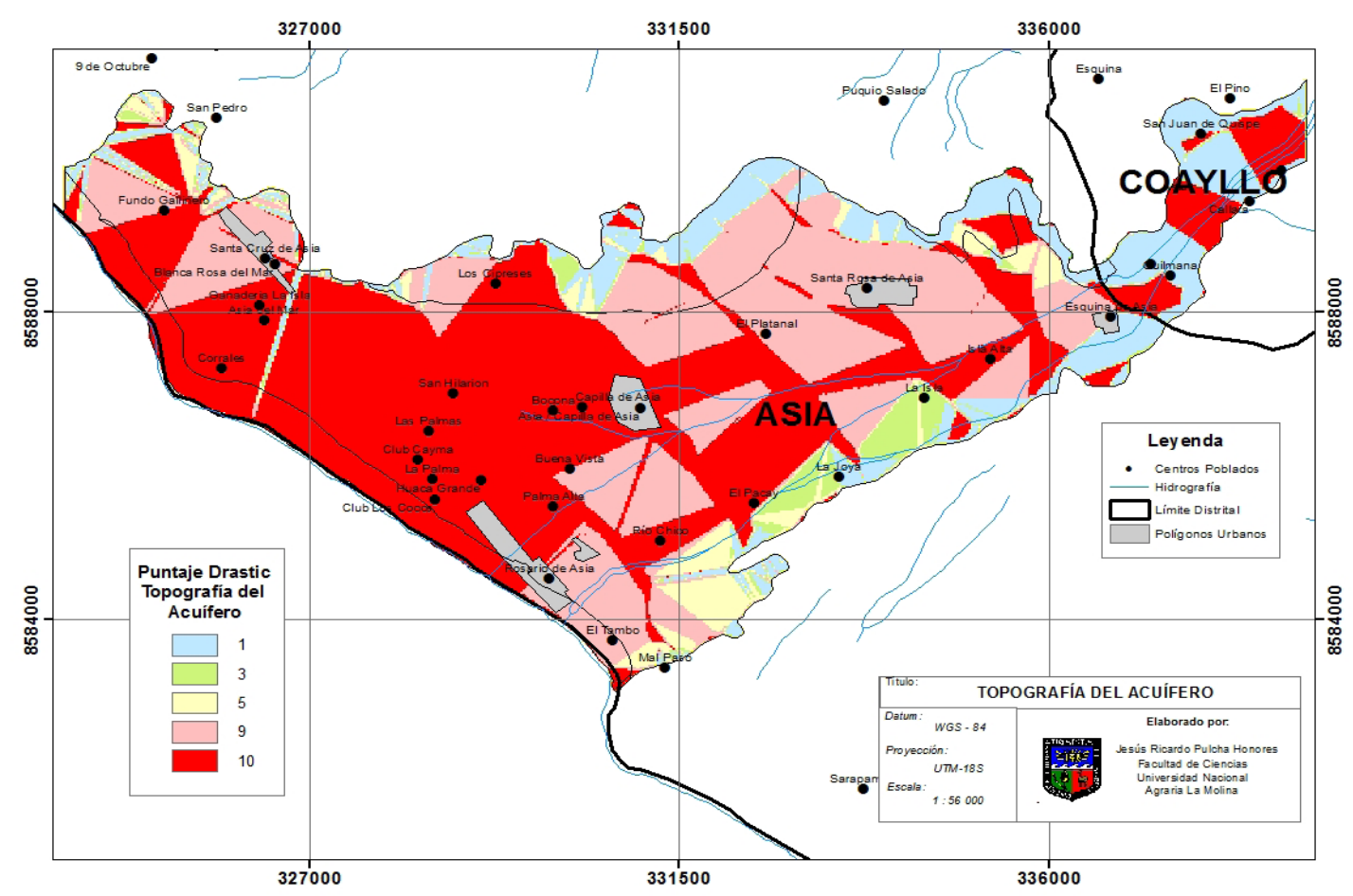

Figura 3. Reclasificación Espacial de Valores de Pendiente del Acuífero.

Según las 8 pruebas de conductividad hidráulica realizadas en el distrito de Asia, tenemos que la permeabilidad o conductividad hidráulica en el acuífero, varía entre 7.69 y $116.12 \mathrm{~m} /$ día (ANA, 2002). Los pozos escogidos para la realización de estas pruebas fueron aquellos que presentaban condiciones técnicas mínimas para su ejecución (ANA, 2011). Al no existir un criterio de distribución espacial de estas pruebas, se optó por colocar el puntaje de máxima vulnerabilidad intrínseca de 10.

\subsection{Determinación de los parámetros del índice DRASTIC SEA}

Los 5parámetros hidrogeológicos que permiten obtener el índice DRASTIC SEAson:

\section{a. Distancia a la línea de costa}

Para la elaboración del mapa de distancias a la línea de costa, se digitalizó la línea de costa del Acuífero AsiaOmas, luego con la herramienta Euclidean Distance del módulo Análisis Espacial del Arcgis10 y utilizando como límite el área de estudio se procedió a calcular las distancias entre píxeles y reclasificar el mapa de pendientes, según los valores Drastic SEA DINDEX, tal como se muestra en la tabla 4 y la Fig. 4.

\section{b. Litología del acuífero}

En la tabla 5 y Fig. 5 se muestra la reclasificación de las zonas geológicas identificadas según los valores Drastic SEA DINDEX.

\section{c. Conductividad hidráulica}

Debido a que no se cuenta con información de conductividad hidráulica en los pozos en la zona de estudio se asignó un puntaje máximo DRASTIC SEA DINDEX igual a 5 en concordancia con lo especificado en el mismo apartado para el índice Drastic general.

\section{d. Zona saturada}

Según los perfiles litológicos IRHS N ${ }^{\circ}$ 15/05/02 - 50 y IRHS $\mathrm{N}^{\circ} 15 / 05 / 02-66$, realizados por la Autoridad Nacional del Agua, la extensión de la Zona Saturada es de $70 \mathrm{~m}$ y la altura máxima del techo de la napa freática es de $44.27 \mathrm{~m}$, por lo que se le asignó un puntaje Drastic SEA - DINDEX de igual a 3.

Tabla 4. Reclasificación de Valores de Distancias a la Línea de Costa.

\begin{tabular}{cc}
\hline Distancia $(\mathrm{km})$ & Puntaje \\
\hline $0-0.5$ & 5 \\
$0.5-1.5$ & 4 \\
$1.5-3.0$ & 3 \\
$3.0-6.0$ & 2 \\
$>6.0$ & 1 \\
\hline
\end{tabular}

Tabla 5. Reclasificación de Valores de Litología del Acuífero.

\begin{tabular}{cc}
\hline Litologia & Puntaje \\
\hline Depósitos Marinos (Qr-m) & 5 \\
Depósitos Aluviales (Qr-al ) & 4 \\
Grupo del Morro del Solar (Ki -ms). & 3 \\
Depósitos Eólicos (Qr-e) & 1 \\
\hline
\end{tabular}




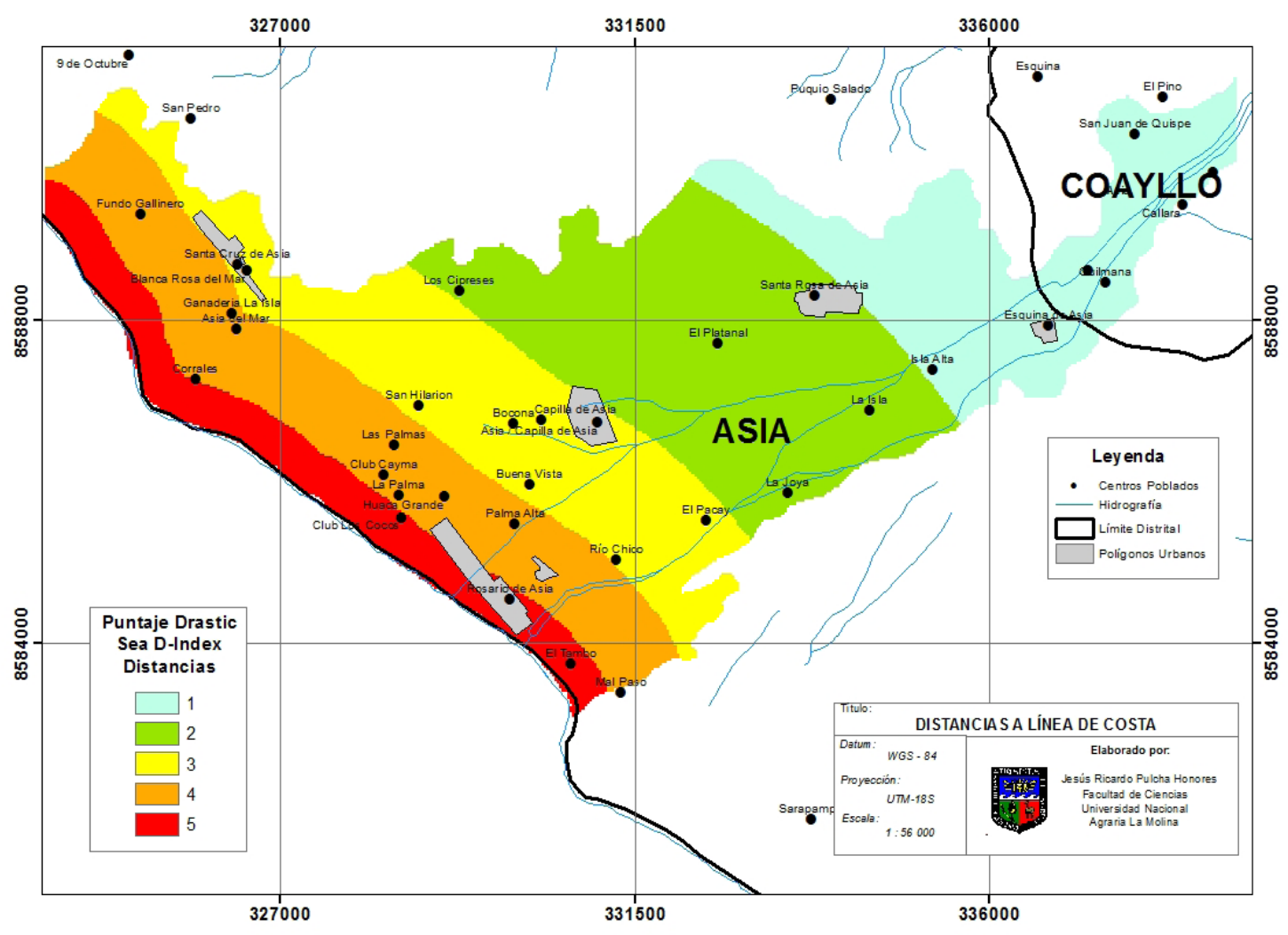

Figura 4. Reclasificación Espacial de Distancias a Línea de Costa.

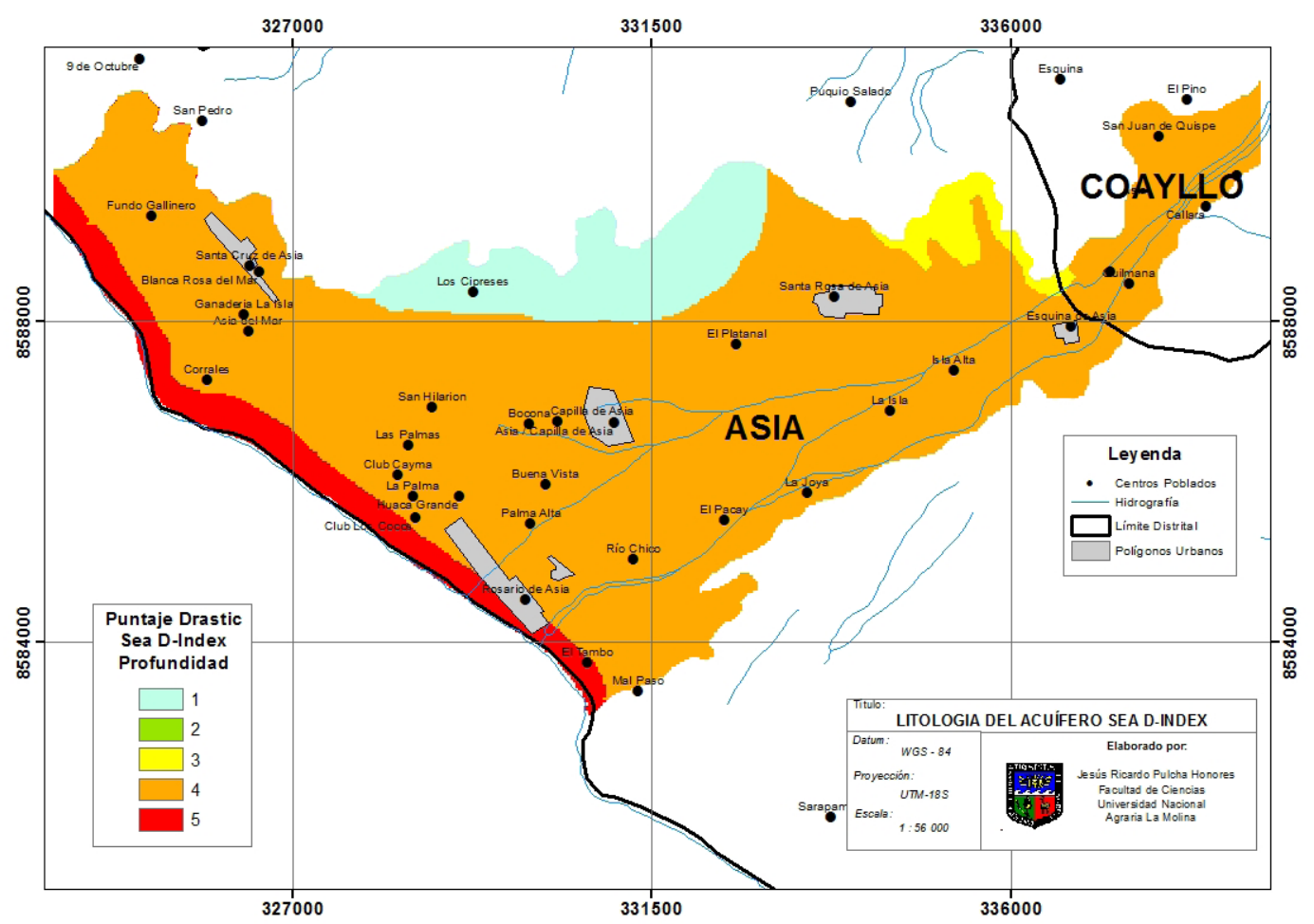

Figura 5. Reclasificación Espacial de Litología del Acuífero. 


\section{e. Nivel piezométrico}

Se realizó la reclasificación de los niveles piezométricos según los valores Drastic SEA DINDEX, la misma que se muestran en la tabla 6 y fig. 6 .

Tabla 6. Reclasificación de Valores del Nivel Piezométrico Drastic Sea DIndex

\begin{tabular}{cc}
\hline Distancia (km) & Puntaje \\
\hline $0-0.5$ & 5 \\
$0.5-1.5$ & 4 \\
$1.5-3.0$ & 3 \\
$3.0-6.0$ & 2 \\
$>6.0$ & 1 \\
\hline
\end{tabular}

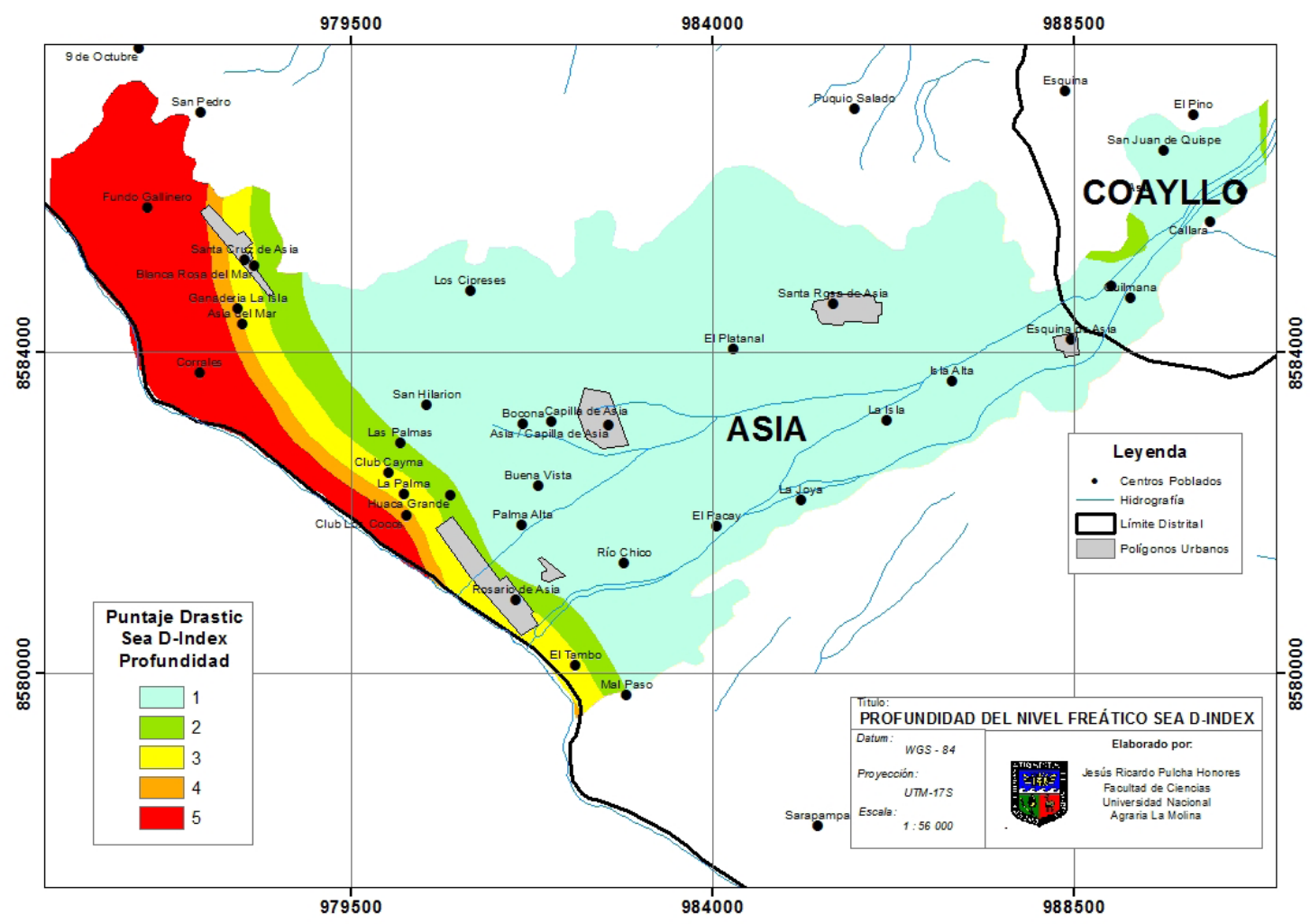

Figura 6. Reclasificación Espacial de Profundidad del Nivel Freático Sea D-Index.

2.4 Elaboración de los mapas de vulnerabilidad Drastic, Drastic Pesticida y Drastic Sea Dindex

A partir de las reclasificación de cada uno de los parámetros según los puntajes Drastic, se procedió a realizar la superposición cartográfica y algebra de mapas mediante el Software Arcgis 10, utilizando la herramienta Raster Calculator. El álgebra de Mapas se realizó utilizando los siguientes Algoritmos para cada uno de los índices.

INDICE DRASTIC y PESTICIDA:

Índice de Vulnerabilidad $=\mathrm{D}_{\mathrm{R}} \mathrm{D}_{\mathrm{W}}+\mathrm{R}_{\mathrm{R}} \mathrm{R}_{\mathrm{W}}+\mathrm{A}_{\mathrm{R}} \mathrm{A}_{\mathrm{W}}+$ $\mathrm{S}_{\mathrm{R}} \mathrm{S}_{\mathrm{W}}+\mathrm{T}_{\mathrm{R}} \mathrm{T}_{\mathrm{W}}+\mathrm{I}_{\mathrm{R}} \mathrm{I}_{\mathrm{W}}+\mathrm{C}_{\mathrm{R}} \mathrm{C}_{\mathrm{W}}$

\section{INDICE SEA DINDEX}

Índice de Vulnerabilidad a la intrusión salina $=\mathrm{L}_{\mathrm{R}} \mathrm{L}_{\mathrm{W}}+$ $\mathrm{A}_{\mathrm{R}} \mathrm{A}_{\mathrm{W}}+\mathrm{Z}_{\mathrm{R}} \mathrm{Z}_{\mathrm{W}}+\mathrm{C}_{\mathrm{R}} \mathrm{C}_{\mathrm{W}}+\mathrm{P}_{\mathrm{R}} \mathrm{P}_{\mathrm{W}}$

Dónde: los subíndices $\mathrm{R}$ y W son el puntaje (rating) y el peso de ponderación (weight) respectivamente. En la tabla 7 se muestran los pesosde ponderación de los parámetros de los 3 índices.
Tabla 7. Peso de ponderación de los parámetros del índice Drastic.

\begin{tabular}{lccc}
\hline Parámetros & $\begin{array}{c}\text { Ponderación } \\
\text { DRASTIC }\end{array}$ & $\begin{array}{c}\text { Ponderación } \\
\text { PESTICIDA }\end{array}$ & $\begin{array}{c}\text { Ponderación } \\
\text { SEA }\end{array}$ \\
\hline $\begin{array}{l}\text { D: Profundidad } \\
\text { hasta el nivel } \\
\text { freático }\end{array}$ & 5 & 5 & - \\
$\begin{array}{l}\text { R: Recarga neta } \\
\text { A: Naturaleza }\end{array}$ & 4 & 4 & - \\
$\begin{array}{l}\text { litológica del } \\
\text { acuífero }\end{array}$ & 3 & 3 & 1 \\
$\begin{array}{l}\text { S: Tipo de suelo } \\
\text { en el acuífero }\end{array}$ & 2 & 5 & - \\
$\begin{array}{l}\text { T: Topografía } \\
\text { I: Impacto de la } \\
\text { zona vadosa }\end{array}$ & 1 & 3 & - \\
$\begin{array}{l}\text { : Conductividad } \\
\text { hidráulica }\end{array}$ & 5 & 4 & - \\
$\begin{array}{l}\text { L: Distancia a la } \\
\text { línea de costa }\end{array}$ & - & 2 & 2 \\
$\begin{array}{l}Z: \text { Zona saturada } \\
\text { P: Nivel } \\
\text { piezométrico }\end{array}$ & - & - & 3 \\
\hline
\end{tabular}




\section{Resultados y discusiones}

\section{1 Índice DRASTIC}

A partir de la superposición cartográfica o álgebra de mapas se generó el Mapa de Vulnerabilidad Intrínseca del Acuífero Asia-Omas a la Contaminación del Agua Subterránea según los parámetros del Índice Drastic (Ver Fig. 7), identificándose y cuantificándose las áreas según su nivel de vulnerabilidad. (Ver tabla 8).

Tabla 8. Porcentaje de áreas de vulnerabilidad intrínseca a la Contaminación de las aguas subterráneas en el Acuífero Asia-Omas.

\begin{tabular}{cccc}
\hline Vulnerabilidad & Índice & Área (ha) & $\%$ Área Total \\
\hline Despreciable & $95-100$ & 80,99 & 1,63 \\
Muy Baja & $101-119$ & 1158,58 & 23,29 \\
Baja & $120-139$ & 2023,64 & 40,68 \\
Moderada & $140-159$ & 1468,07 & 29,51 \\
Alta & $160-164$ & 242,93 & 4,88 \\
\hline
\end{tabular}

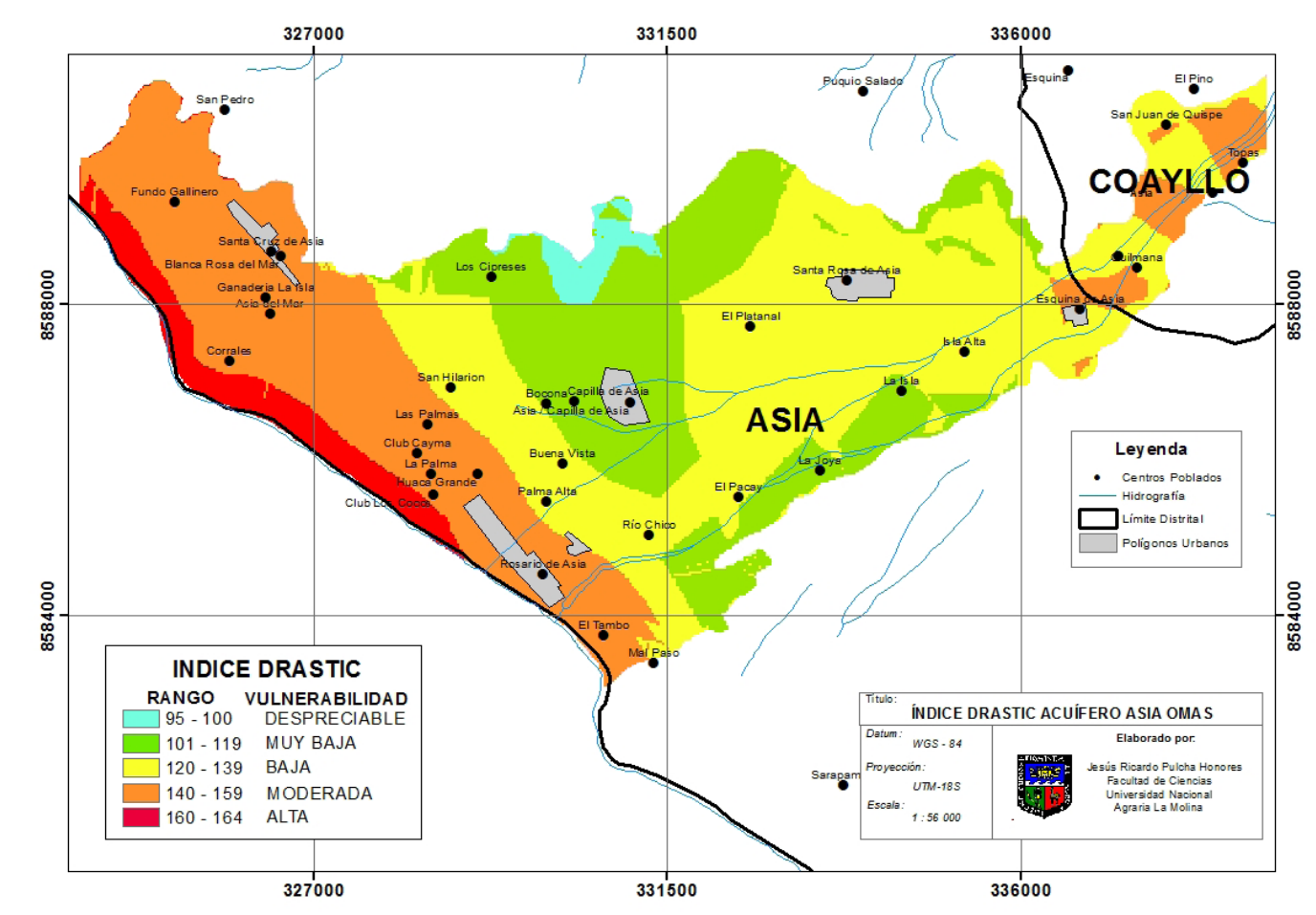

Figura 7. Mapa de Vulnerabilidad Intrínseca a la Contaminación del Agua Subterránea del Acuífero Asia-Omas.

El valor estimado promedio para el índice DRASTIC fue de 130. El valor mínimo obtenido fue de 95 y valor máximo alcanzado fue de 164.

En la Fig. 7 se puede observar que las zonas de vulnerabilidad baja y muy baja se distribuyen en el área media del acuífero, mientras que las zonas con vulnerabilidad moderada y alta se encuentran ubicadas en la parte baja del mismo.

Las variables de profundidad del agua subterránea, recarga y zonda vadosa, son las más influyentes para este indicador de la distribución de la vulnerabilidad en el área del acuífero. Debido a esto, es lógico el resultado obtenido de mayores áreas con baja vulnerabilidad, ya que al factor Recarga se le asignó el valor mínimo, debido a la baja y /o inexistencias de precipitaciones en la zona de estudio.

En la tabla 8, se observa que para el índice DRASTIC, el acuífero Asia-Omas se ha clasificado con categorías de vulnerabilidad muy baja, baja y moderada que representan el $91.48 \%$ del área total del acuífero.

Ninguna zona del acuífero fue ubicada en el rango de vulnerabilidad Muy alta, sin embargo un 33\% del área del acuífero se encuentra en zonas vulnerabilidad moderada a alta evidenciando la necesidad de normar adecuadamente el desarrollo de actividades en la zona con el objetivo de mantener la protección de los acuíferos.

\section{2 Índice DRASTIC PESTICIDA}

De acuerdo a los parámetros del Índice Drastic y los pesos asignados de vulnerabilidad a Pesticidas, se generó el Mapa de Vulnerabilidad a la Contaminación por Pesticidas del Agua Subterránea en el Acuífero AsiaOmas (Ver Fig. 8). En la tabla 9 se cuantifican las áreas del acuífero según su nivel de vulnerabilidad a pesticidas. El valor promedio estimado para el índice DRASTIC PESTICIDA fue de 109. El valor mínimo obtenido fue de 65 y valor máximo alcanzado fue de 152 .

El índice de vulnerabilidad DRASTIC PESTICIDA, no mostró zonas clasificadas con Vulnerabilidad Alta o Muy Alta. En su mayor parte el área del acuífero fue clasificada en el rango de vulnerabilidad Muy Baja 
(41.24\%), siendo clasificada el área de mayor riesgo a la contaminación por pesticidas con una vulnerabilidad Moderada, representando un $21.29 \%$.

En la determinación del índice DRASTIC PESTICIDA, los parámetros que presentan mayor influencia son profundidad del agua subterránea, recarga y tipo de suelo. Es por esta razón que los resultados obtenidos presentan niveles de vulnerabilidad bajos, debido a que se asignaron valores mínimos a los parámetros recarga y tipo de suelo.

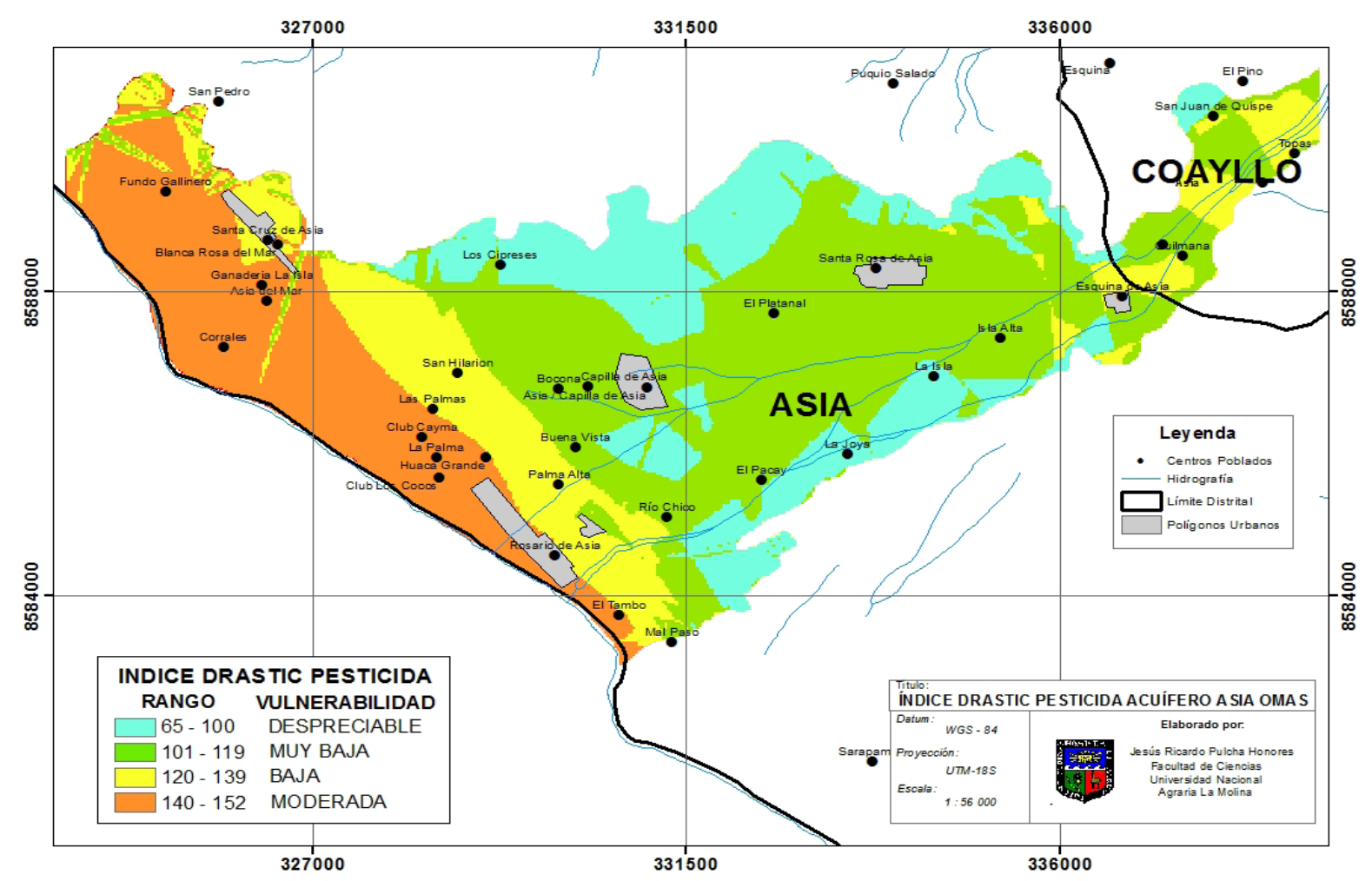

Figura 8. Mapa de Vulnerabilidad a la Contaminación por Pesticidas del Agua Subterránea en el Acuífero Asia-Omas.

Tabla 9. Porcentaje de Áreas vulnerables a la Contaminación por Pesticidas del Agua Subterránea en el Acuífero Asia-Omas.

\begin{tabular}{cccc}
\hline Vulnerabilidad & $\begin{array}{c}\text { Índice } \\
\text { DRASTIC }\end{array}$ & Área (ha) & $\begin{array}{c}\text { \% Área } \\
\text { Total }\end{array}$ \\
\hline Despreciable & $65-100$ & 1010,07 & 20,31 \\
Muy Baja & $101-119$ & 2051,27 & 41,24 \\
Baja & $120-139$ & 853,71 & 17,16 \\
Moderada & $140-152$ & 1059,21 & 21,29 \\
\hline
\end{tabular}

\subsection{INDICE DRASTIC - SEA DINDEX}

En la Fig. 9 se muestra el Mapa de Vulnerabilidad a la Intrusión Salina en el Acuífero Asia-Omas y en la tabla 10 se cuantifican las áreas según su nivel de vulnerabilidad. El valor promedio estimado para el índice DRASTIC SEA INDEX fue de 31. El valor mínimo obtenido fue de 19 y valor máximo alcanzado fue de 43 .

En la tabla 10, se observa que para el índice DRASTIC SEA INDEX se clasificaron las áreas del acuífero en 5 categorías que van desde Despreciable a Alta, siendo las categorías de vulnerabilidad baja y despreciable las más representativas (79\% del área del acuífero), y las categorías de vulnerabilidad moderada y alta los menos representativas $(21 \%)$.

En la figura 9 se puede observar que las zonas de mayor vulnerabilidad a la intrusión salina se ubican lógicamente en las partes bajas del acuífero y paralela a la línea de costa, disminuyendo la vulnerabilidad mientras más nos alejamos a la línea de costa.

Para el índice Drastic Sea Índex los parámetros de distancia a línea de costa y conductividad hidráulicason las más influyentes para la intrusión Salina, es por ello que las áreas de alta vulnerabilidad se ubican en las zonas cercanas al litoral costero.

Tabla 10. Porcentaje de áreas de Vulnerabilidad a la Contaminación por Agua de Mar debido al Proceso de Intrusión Salina.

\begin{tabular}{cccc}
\hline Vulnerabilidad & $\begin{array}{c}\text { Índice } \\
\text { DRASTIC }\end{array}$ & Área (ha) & $\begin{array}{c}\text { \% Área } \\
\text { Total }\end{array}$ \\
\hline Despreciable & $19-24$ & 1341.22 & 26.84 \\
Muy Baja & $24-29$ & 1972.28 & 39.47 \\
Baja & $29-33$ & 636.83 & 12.74 \\
Moderada & $33-38$ & 309.56 & 6.19 \\
Alta & $38-43$ & 737.17 & 14.75 \\
\hline
\end{tabular}




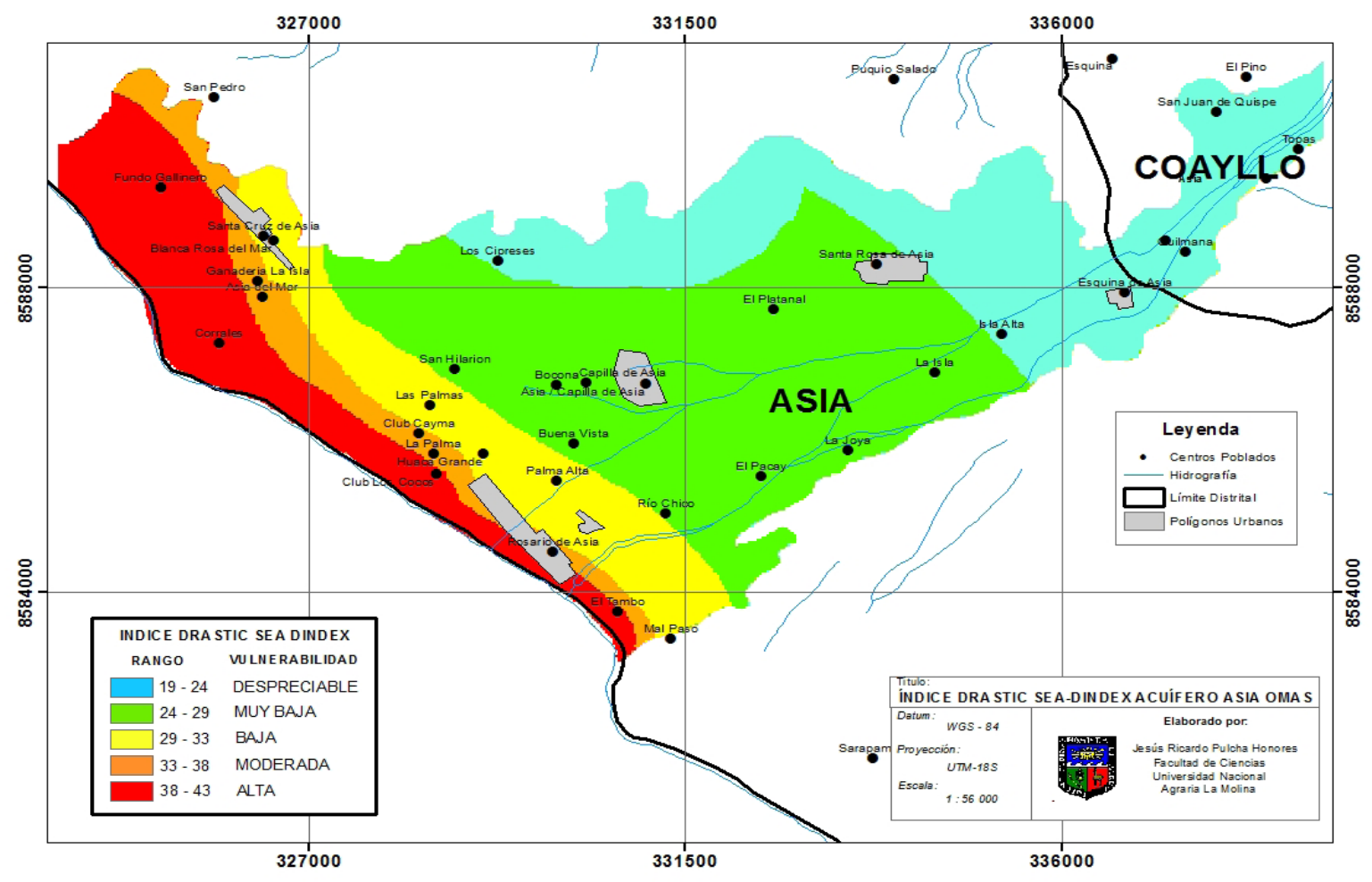

Figura 9. Mapa de Vulnerabilidad a la Intrusión Salina en el Acuífero Asia-Omas.

\section{Conclusiones}

El grado de penetración del contaminante en el área del acuífero es bajo, debido a que los resultados de los índices DRASTIC identificaron en su mayor proporción zonas con vulnerabilidad baja, sin embargo la categoría Moderada alcanzó un 29.5\%, del área de estudio; por lo que las medidas de prevención y gestión del agua se deben priorizar en esta zona.

Los resultados del Índice DRASTIC SEA DINDEX, identifican zonas con alta y moderada vulnerabilidad a la intrusión salina, por lo que es en estas zonas, que se debe prestar mayor atención en el control de la explotación del recurso hídrico subterráneo, ya que el proceso de intrusión salina puede ser acelerado, debido al alto potencial agrícola y crecimiento urbano desordenado en la zona.

Los resultados del índice DRASTIC PESTICIDA indican que en el área de estudio se tiene una predominancia de la Vulnerabilidad muy Baja. Este resultado evidencia que el acuífero presenta una buena capacidad natural de atenuación a este tipo específico de contaminantes.

Para mejorar la precisión en el cálculo del índice DRASTIC e implementarse como una herramienta importante para la Gestión Ambiental del Recursos Hídrico Subterránea y el Ordenamiento Territorial del acuífero Asia-Omas, es necesario generar información cartográfica actualizada y realizar monitoreo continuo de los pozos de explotación en la zona de estudio.

\section{Literatura citada}

Aller, L.; Lerh, J.; Petty, R.; Hac, G. and Drastric. 1987. a standardized system for evaluating groundwater pollution potential using hydrogeologic settings. s.e. EPA-600/2-87-0305. p. 1987.

ANA (Autoridad Nacional del Agua). 2011. Evaluación de las Aguas Subterráneas del Acuífero Asia Omas. Lima. 117 p. 2011.

ANA (Autoridad Nacional del Agua). 2002. Inventario y Monitoreo de las Aguas Subterráneas del Valle Asia Omas. Lima. 121 p. 2002.

Bericiartua, P. J. 2003. Vulnerabilidad de Aguas Subterráneas a la Contaminación. Buenos Aires. 20 p. 2003.

Bocanegra, E. M.; Massone, H.; Bernasconi, M. V. \&Vrba, J. 2004. Methodological approach for the assessment of the vulnerability of coastal aquifers. International conference on groundwater vulnerability assessment and mapping. Ustron. Poland. ISBN 8387431-60-5. Procedings:33-34. 2004.

EPA (Enviromental Protection Agency). 1991. A review of methods for assessing the sensitivity of aquifers to pesticide contamination. Preliminary document. Washington: EPA, 1, pp. 1-21. 1991.

INGEMMET (Instituto Geológico Minero Metalúrgico). 1993. Geología de los Cuadrángulos de Mala, Lunahuana, Tupe, Chincha, Tantara y Castrovirreyna, Lima, 1993. 
Losilla, M.; Rodríguez, H.; Schosinsky, G., Stimson, J. y Bethune, D. 2001. Los acuíferos volcánicos y el desarrollo sostenible en América Central. Editorial de la Universidad de Costa Rica. 205 p. 2001. 